\title{
Discovery and characterization of a novel class of pyrazolopyrimidinedione tRNA synthesis inhibitors
}

\author{
Justin I Montgomery ${ }^{1}$, James F Smith ${ }^{2}$, Andrew P Tomaras ${ }^{2,3}$, Richard Zaniewski ${ }^{2}$, Craig J McPherson ${ }^{2}$, \\ Laura A McAllister ${ }^{1}$, Sandra Hartman-Neumann ${ }^{2}$, Joel T Arcari ${ }^{1}$, Marykay Lescoe ${ }^{2}$, Jemy Gutierrez ${ }^{2,3}$, \\ Ying Yuan ${ }^{2,4}$, Chris Limberakis ${ }^{1}$ and Alita A Miller ${ }^{25}$
}

A high-throughput phenotypic screen for novel antibacterial agents led to the discovery of a novel pyrazolopyrimidinedione, PPD-1, with preferential activity against methicillin-resistant Staphylococcus aureus (MRSA). Resistance mapping revealed the likely target of inhibition to be lysyl tRNA synthetase (LysRS). Preliminary structure-activity relationship (SAR) studies led to an analog, PPD-2, which gained Gram-negative antibacterial activity at the expense of MRSA activity and resistance to this compound mapped to prolyl tRNA synthetase (ProRS). These targets of inhibition were confirmed in vitro, with PPD-1 showing $\mathrm{IC}_{50} \mathrm{~S}$ of 21.7 and $35 \mu \mathrm{m}$ in purified LysRS and ProRS enzyme assays, and PPD-2, 151 and $0.04 \mu \mathrm{m}$, respectively. The highly attractive chemical properties of these compounds combined with intriguing preliminary SAR suggest that further exploration of this compelling novel series is warranted.

The Journal of Antibiotics (2015) 68, 361-367; doi:10.1038/ja.2014.163; published online 3 December 2014

\section{INTRODUCTION}

The importance of identifying new antibacterial agents employing novel mechanisms of action cannot be overstated. Bacterial resistance to currently available therapeutic agents continues to be a serious and growing problem, with calls for action now being made routinely by both private and public sectors, including the Centers for Disease Control and the World Health Organization. ${ }^{1-4}$ In the search for novel compounds, it is important to remember that nearly every known antibiotic class was first identified by its ability to kill bacteria; in some cases, the mode of action was not determined until many years later. ${ }^{5}$ In contrast, target-based screening strategies to identify inhibitors of essential proteins have been less successful in generating novel antibiotics with desirable attributes. ${ }^{6}$ Although potent inhibitors of a number of attractive targets have been identified with this approach, their development into drugs has often been hindered by the inability of medicinal chemistry to 'design in' bacterial cell penetration. Accordingly, a simple phenotypic screen for inhibition of bacterial growth was conducted to identify compounds, which were preselected for their promising physical chemical properties, for whole-cell antibacterial activity; the mode of action of hit compounds was subsequently determined using a 'reverse genomics' platform that combined several biochemical and genetic approaches. ${ }^{7}$ The following describes the discovery and characterization of one such compound, PPD-1, a novel pyrazolopyrimidinedione with anti-methicillin-resistant Staphylococcus aureus activity. PPD-1 was found to inhibit bacterial translation at the tRNA synthesis step. Preliminary structure-activity relationship studies led to an analog, PPD-2, whose antibacterial spectrum was found to be distinct from the parent compound, which correlated to changes in target specificity. The highly attractive chemical properties of these compounds, including potential for extensive medicinal chemistry exploration, are fairly unusual at this stage of discovery. This fact, combined with intriguing preliminary structure-activity relationship results, suggests that further characterization of the PPD series may be warranted as part of the global search for novel antibiotics.

\section{MATERIALS AND METHODS}

Whole-cell antibacterial high throughput screen

Single-use frozen stocks of the $P$. aeruginosa lab strain wild-type PAO1 (American Type Culture Collection, Manassas, VA, USA) were thawed and diluted to $1 \times 10^{5} \mathrm{CFU} \mathrm{m}{ }^{-1}$ in Mueller Hinton Broth. Twenty $\mu$ l was then added to individual wells of 384-well Costar plates (catalog \#3701, VWR, Radnor, PA, USA) with each well containing $0.5 \mu$ l of test compound at $2 \mathrm{~mm}$ in dimethyl sulfoxide (DMSO), resulting in a final screening concentration of $97 \mu \mathrm{M}$ in $2.4 \%$ DMSO. Plates were covered and placed in a humidified $37^{\circ} \mathrm{C}$ incubator for 18-22 h, after which the $\mathrm{OD}_{620 \mathrm{~nm}}$ was determined using a Spectramax multiscan plate reader (Molecular Devices, Sunnyvale, CA, USA). Any well with an $\mathrm{OD}_{620 \mathrm{~nm}}$ value of $<30 \%$ that of growth control wells (containing no compound) was considered a 'hit'. Approximately 1.3 million compounds

${ }^{1}$ Department of Medicinal Chemistry, Antibacterials Research Unit, Pfizer Worldwide Research and Development, Groton, CT, USA and ${ }^{2}$ Department of Discovery Biology, Antibacterials Research Unit, Pfizer Worldwide Research and Development, Groton, CT, USA

${ }^{3}$ Current address: Pfizer Global Biotherapeutics, Cambridge, MA, USA.

${ }^{4}$ Current address: Global Therapeutics Research, Zoetis, Kalamazoo, MI, USA.

${ }^{5}$ Current address: AstraZeneca Infection Innovative Medicines, Waltham, MA, USA.

Correspondence: Dr AA Miller, AstraZeneca Infection Innovative Medicines, 35 Gatehouse Drive, Waltham, MA 02451, USA.

E-mail: alita.miller@astrazeneca.com

Received 28 July 2014; revised 27 October 2014; accepted 2 November 2014; published online 3 December 2014 
proprietary to Pfizer were screened, resulting in an average $Z$-value of 0.81 and a hit rate of $\sim 0.02 \%$. Hit confirmation and preliminary assessment of the spectrum were performed by testing each compound for activity over eight twofold-concentration dilutions against the following common laboratory strains in the same manner as described above: Pseudomonas aeruginosa (PAO1), Staphylococcus aureus (RN4220), Klebsiella pneumonaie (KP3700) and Acinetobacter baumannii (AB3176).

\section{MIC determination}

The MIC necessary to inhibit bacterial growth was determined for compounds of interest by broth microdilution according to standard protocols. ${ }^{8}$

\section{Cytotoxicity assay}

ThlE-2 cells (American Type Culture Collection) were seeded at a density of 2500 cells per well in 384-well clear-bottom tissue culture plates containing $25 \mu \mathrm{l}$ of standard cell culture medium. After $24 \mathrm{~h}$ of incubation at $37^{\circ} \mathrm{C}$ and $5 \%$ $\mathrm{CO}_{2}$, the medium was removed and replaced with $25 \mu \mathrm{l}$ of fresh medium containing appropriately diluted test compounds (ranging from 300 to $0.03 \mu \mathrm{M}$ ). All compounds were initially solubilized in $100 \%$ DMSO and tested in triplicate. The final concentration of DMSO in each well was $1.0 \%$. Following a 72 -h exposure to compounds at $37^{\circ} \mathrm{C}$ and $5 \% \mathrm{CO}_{2}$, cell viability in each well was determined by measuring the concentration of cellular ATP using the Cambrex Vialight Plus Cell Proliferation/Cytoxicity Kit as described by the manufacturer (Lonza, Allendale, NJ, USA). ATP concentration was determined by reading luminescence in an Envision plate reader (Perkin Elmer, Waltham, MA, USA). Percent of viable cells relative to untreated controls was determined for each well. Final $\mathrm{IC}_{50}$ value corresponds to the dose projected to kill $50 \%$ of the cells following a 72 -h exposure.

\section{Macromolecular synthesis assay}

Inhibition of specific macromolecular synthesis pathways was assessed by measuring the incorporation of radiolabelled precursors in a growing culture of Eschericia coli JL553 (tolC, lysE) as follows. MOPS-defined media (Teknova, Hollister, CA, USA) was inoculated with an overnight culture of E. coli JL553

\section{a Macromolecular Synthesis Assay}

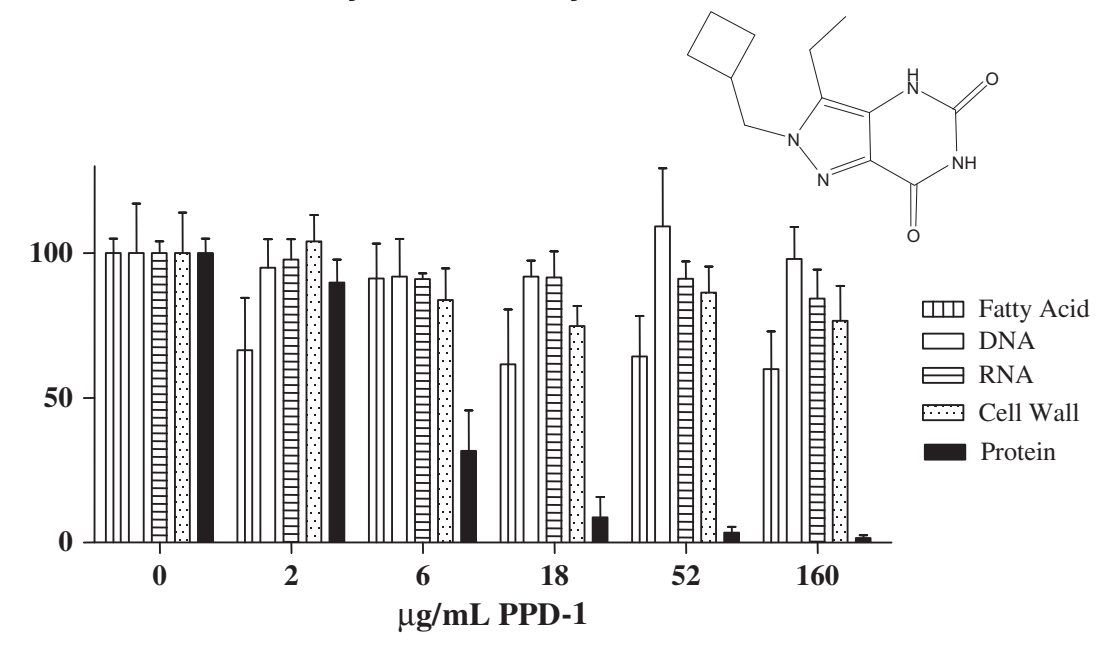

b TnT Staging Assay

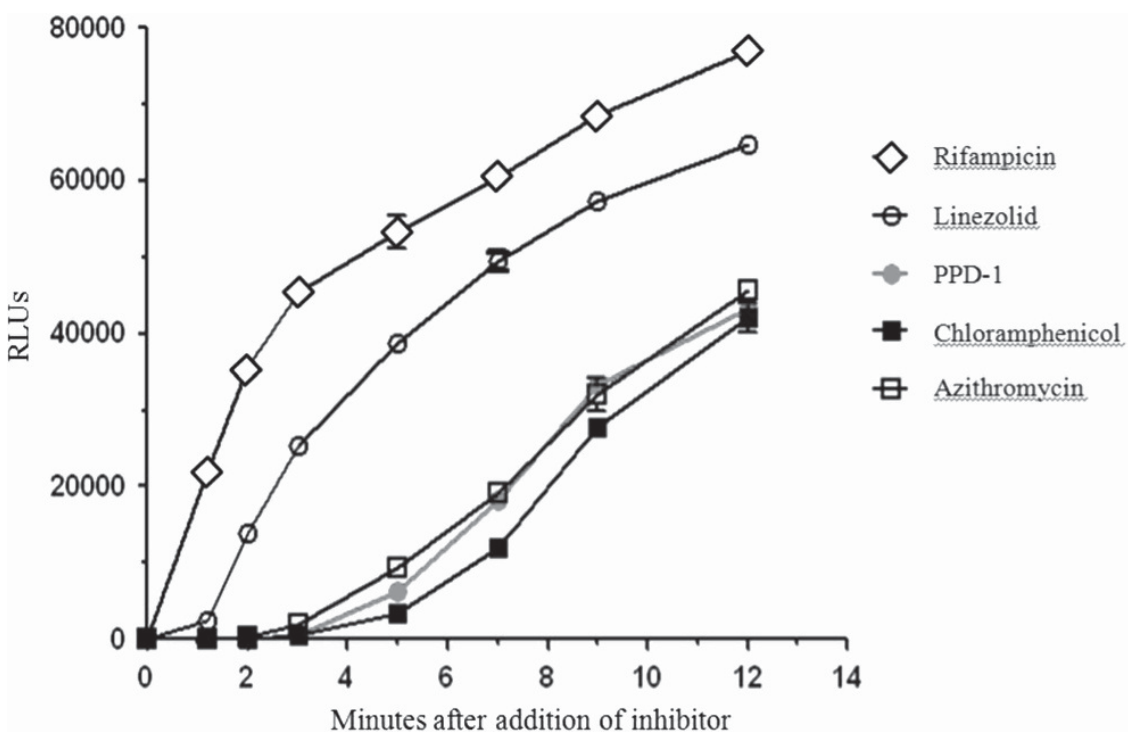

Figure 1 PPD-1 is a protein synthesis inhibitor. (a) E. coli treated with increasing amounts of PPD-1 resulted in a dose-dependent inhibition of incorporation of amino-acid precursors in a macromolecular synthesis assay ( $\mathrm{Y}$ axis corresponds to percent of wild-type control). Inset shows the structure of PPD-1. (b) PPD-1 was compared with known RNA and protein synthesis inhibitors in a transcription-translation (TnT) staging assay to assess the point at which inhibition likely occurs. Activity is shown as relative luminescence units (RLUs). A full color version of this figure is available at The Journal of Antibiotics journal online. 
and incubated at 200 r.p.m. at $37^{\circ} \mathrm{C}$ to an $\mathrm{OD}_{600}$ of $0.4-0.5$. A $100-\mu \mathrm{l}$ aliquot of this culture was added to a 96-well plate containing $5 \mu$ of antibiotic stocks to result in a final concentration range of $0.5-20 \times$ the MIC and incubated for $10 \mathrm{~min}$ with gentle shaking at $35^{\circ} \mathrm{C}$. Ten $\mu \mathrm{l}$ of ${ }^{14} \mathrm{C}$-radiolabelled precursors for each macromolecular pathway were added as follows: DNA, 1:4 dilution of thymidine $\left(50 \mu \mathrm{Ci} \mathrm{ml}^{-1}\right.$, Amersham, GE Healthcare, Pittsburgh, PA, USA, cat \# CFA-219); RNA, 1:100 dilution of uridine $\left(50 \mu \mathrm{Ci} \mathrm{ml}^{-1}\right.$, Amersham cat \# CFB-51); protein, 1:55 dilution of l-leucine $\left(50 \mu \mathrm{Ci} \mathrm{ml}^{-1}\right.$, Amersham cat \# CFB-183); fatty acid, 1:20 dilution of sodium acetate $\left(200 \mu \mathrm{Ci} \mathrm{ml}^{-1}\right.$, Amersham cat \# CFA-14) and cell wall, 1:20 dilution of diaminopimelic acid (1000 $\mu \mathrm{Ci} \mathrm{ml}^{-1}$, Moravek Biochemicals, Brea, CA, USA, cat \# MT-1556). Plates were incubated for an additional $25 \mathrm{~min}$ at $35^{\circ} \mathrm{C}$ with gentle shaking. Following incubation, $100 \mu$ of ice-cold $25 \%$ trichloroacetic acid was added to each well, and the plates were incubated on ice for $1 \mathrm{~h}$. Incorporated counts were harvested with the Packard FilterMate-96 harvester (Perkin Elmer) using 'UniFilter GF/B' filter plates (Perkin Elmer). The filter plates were prewashed with $5 \%$ trichloroacetic acid, samples were filtered through and then washed twice with ice-cold 5\% trichloroacetic acid. A final wash with $10 \%$ ethanol was performed, and the plates dried under a heat lamp for $5 \mathrm{~min}$ and then allowed to dry for an additional $\sim 10 \mathrm{~min}$ at room temperature. The bottoms of the plates were sealed, and $40 \mu \mathrm{l}$ of MicroScint scintillation fluid (PerkinElmer) was added per well. The plates were then top-sealed with MultiScreen sealing tape (Thermo Fisher, Rockville, MD, USA) and counted on the TopCount (PerkinElmer), 1 min per well. Experiments were conducted at least in triplicate. Data analysis was performed using GraphPad Prism (La Jolla, CA, USA).

\section{Transcription-translation ( $\mathrm{Tn} T)$ assay}

Inhibitors of bacterial protein synthesis were identified and characterized by their ability to inhibit the production of functional luciferase in a cell-free coupled transcription and translation system. The assay system employs plasmid DNA containing the firefly luciferase gene, an S30 fraction (E. coli cytosol enzymes and ribosomes) and a buffer mix containing necessary transcription and translation reagents and cofactors. Although this assay is commercially available, the results described here were generated using reagents produced in-house, as previously described, ${ }^{9}$ with the following modifications: E. coli BL-21(DE3) grown in M9 minimal media was used to generate S30 fractions and E. coli JM109/pBestLuc plasmid grown in LB media was used for plasmid maintenance and purification. Where indicated, increasing amounts of excess lysine or proline were added up to $150 \mu \mathrm{M}$. Each compound was tested in a 10-point, 1:2 serial dilution format starting at $40 \mu \mathrm{m}$ and diluting down to $0.078 \mu \mathrm{M}$, in at least duplicate.

\section{Staging assay}

The staging assay is a modification of the TnT assay, where excess inhibitor is added at various times during the incubation of a normal $\mathrm{TnT}$ assay and the amount of reporter enzyme is determined at the end of incubation. The activity of different types of inhibitors (transcription, translation initiation, translation elongation, reporter enzyme inhibition, etc.) can be discerned when reporter activity is graphed vs inhibitor time of addition, because the earlier an inhibitor acts in the protein synthesis process, the greater the amounts of enzyme that are present at the end of a given incubation period (Figure 1b). Exploratory compounds and known inhibitors were first tested in the TnT assay described above to generate an $\mathrm{IC}_{50}$ value. The compounds were then diluted with an appropriate volume of a $\mathrm{H}_{2} \mathrm{O} /$ DMSO mixture to generate a compound concentration of 140 times the $\mathrm{IC}_{50}$ value in $<14 \%$ DMSO (for example, the $\mathrm{IC}_{50}$ of azithromycin in the $\mathrm{TnT}$ assay is $0.19 \mu \mathrm{M}$, so a stock solution ( $14 \mathrm{mM})$ was diluted to $22.4 \mu \mathrm{M}$ in $13 \% \mathrm{DMSO})$. This concentration corresponded to a final azithromycin concentration of $3.2 \mu \mathrm{M}$ during the staging assay (that is, 20 times the $\mathrm{IC}_{50}$ value), when $5 \mu \mathrm{l}$ of compound was added to a final assay volume of $35 \mu$ l. Five $\mu \mathrm{l}$ of $0.2 \mathrm{mg} \mathrm{ml}^{-1}$ pBestLuc plasmid ( $1 \mu \mathrm{g}$ of plasmid) was added to each of the 8 wells per compound being assayed in a 96-well plate. Five $\mu$ l of the inhibitor stock was added to well 1 (such that it will result in $20 \times$ the $\mathrm{IC}_{50}$ in the final assay volume) and was used as the time 0 control. Data generated for this well was used to normalize (by subtraction) all the other data points corresponding to increasing times of inhibitor additions.
The reaction was started by adding $25 \mu \mathrm{l}$ of the reaction mix (containing the S30 fraction and extra cofactors as described above) to all the wells. Five $\mu \mathrm{l}$ of inhibitor stock was then added to the other wells at various time points as indicated. After a total of $60 \mathrm{~min}$ of incubation at room temperature, the reaction was stopped with the addition of $35 \mu \mathrm{l}$ of LucLite solution (PerkinElmer) per well. After $7 \mathrm{~min}$ of dark adjustment, the amount of luminescence was read on a Micro-Beta Trilux (Wallach, Sweden). Data were generated in at least duplicate.

\section{Cloning, expression and purification of aminoacyl tRNA synthetases}

For multicopy expression experiments, the lysRS gene from RN4220 was cloned into the xylose-inducible expression vector pHIS1525 and transformed into S. aureus RN4220. To generate purified tRNA synthetases, S. aureus lysRS and E. coli proRS (the latter of which had been amplified from purified E. coli W3110 genomic DNA) were cloned into the expression vector pET15b. N-terminally his-tagged lysyl or prolyl tRNA synthetases (ProRS) were overexpressed upon isopropyl $\beta$-D-1-thiogalactopyranoside induction and purified using standard conditions as described by the manufacturer (Clontech, Mountainview, CA, USA).

\section{Aminoacyl tRNA synthetase assay}

Aminoacyl tRNA synthetase activity was measured in vitro by determining the amount of radiolabelled lysyl or prolyl tRNA that was incorporated over time to a mixture of E. coli tRNAs as follows. First, the following reaction mix was preincubated for $10 \mathrm{~min}$ at room temperature: $50 \mathrm{~mm}$ HEPES, pH 7.6, $10 \mathrm{~mm}$ $\mathrm{MgCl}_{2}, 2 \mathrm{~mm}$ ATP, $10 \mathrm{~mm} \beta \mathrm{ME}, 2 \mathrm{mg} \mathrm{ml}^{-1}$ purified E. coli tRNAs (SigmaAldrich, St Louis, MO, USA), 70 nм purified prolyl or lysysl tRNA synthetase and $0.5 \mu \mathrm{m}$ inhibitor in a total volume of $50 \mu$ l. The reaction was initiated by addition of $0.05 \mu \mathrm{Ci}(3.12 \mu \mathrm{M})\left[\mathrm{C}^{14}\right]$ labeled Proline or Lysine (Amersham). The reaction was carried out at $35^{\circ} \mathrm{C}$ for $20 \mathrm{~min}$ and quenched by addition of $200 \mu \mathrm{l}$ ice-cold ethanol, then placed at $4^{\circ} \mathrm{C}$ for an additional $30 \mathrm{~min}$ to complete precipitation. RNA was filtered through a 96-well GF/B filter plate, which was pre-wet with $10 \mathrm{~mm}$ unlabeled lysine or proline, and the plate was washed twice with cold ethanol to remove unbound radiolabeled substrate. Scintillation fluid (50 $\mu \mathrm{l})$ was added, and ${ }^{14} \mathrm{C}$ counts were measured on the TopCount reader for 120 s. Activity was reported as percent control (for example, number of counts obtained for inhibitor-treated samples as compared with the uninhibited control sample).

\section{Resistance mapping of PPD-1 in Neisseria gonorrhoeae}

An error-prone PCR library using 10-kb PCR amplicons encompassing the entire genome was generated in Neisseria gonorrhoeae strain NG2888 (ATCC) as described. ${ }^{10}$ Briefly, these amplicons were then used as donor DNAs to transform NG2888 in 96-well plates, and cells were plated on 2, 4 and 8 times the MIC. These studies identified two overlapping regions of the genome, both containing lysRS. To identify the mutation responsible for resistance, the overlapping region was PCR-amplified using individual resistant mutants as templates, and the DNA sequence was compared with wild-type NG2888. Finally, these individual amplicons were used as donor DNAs to create isogenic strains containing the mutation of interest.

Resistance mapping of PPD-2 by multicopy suppression in E. coli Spontaneous mutants resistant to PPD-2 were generated using E. coli W3110 tolC::Tn10. Briefly, $2 \times 10^{9}$ cells were plated on cation-adjusted Mueller Hinton Agar plates (Difco, Becton Dickinson, Houston, TX, USA) containing a range of PPD-2 concentrations. Plates were incubated at $37^{\circ} \mathrm{C}$ for $40 \mathrm{~h}$ before counting colonies and calculating resistance frequencies. Genomic DNA from a colony recovered from the $8 \mu \mathrm{g} \mathrm{ml}^{-1}$ PPD-2 plate was extracted, partially digested with Sau3AI and ligated to BamHI-digested pUC19 DNA. The resulting genomic library was electroporated into susceptible E. coli W3110 tolC::Tn10 cells and plated on Mueller Hinton Agar plates containing increasing concentrations of PPD-2. Plasmid DNA from the resulting PPD-2 colonies was extracted and sequenced using M13 primers. 


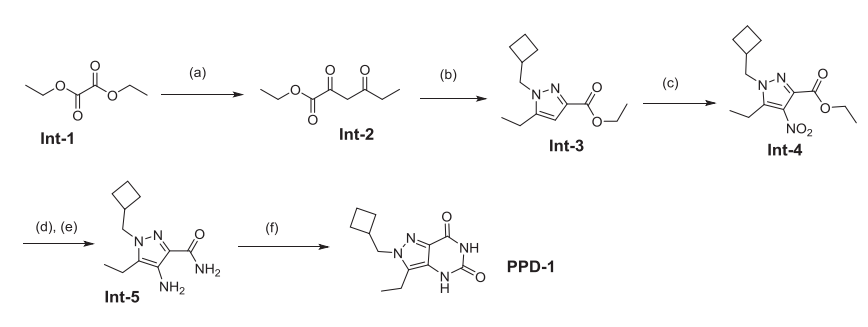

Scheme 1 Synthesis of PPD-1 (a) Methylethyl ketone, $\mathrm{NaOEt} / \mathrm{EtOH}$, reflux; (b) (cyclobutylmethyl)hydrazine, $\mathrm{AcOH}$; (c) $\mathrm{HNO}_{3}, \mathrm{H}_{2} \mathrm{SO}_{4}, 40{ }^{\circ} \mathrm{C}$; (d) $\mathrm{NH}_{3}$, $\mathrm{MeOH}$; (e) $\mathrm{H}_{2}, \mathrm{Pd} / \mathrm{C}, \mathrm{EtOH}$; (f) $\mathrm{CDI}, \mathrm{NEt}_{3}, \mathrm{MeCN}$, reflux.

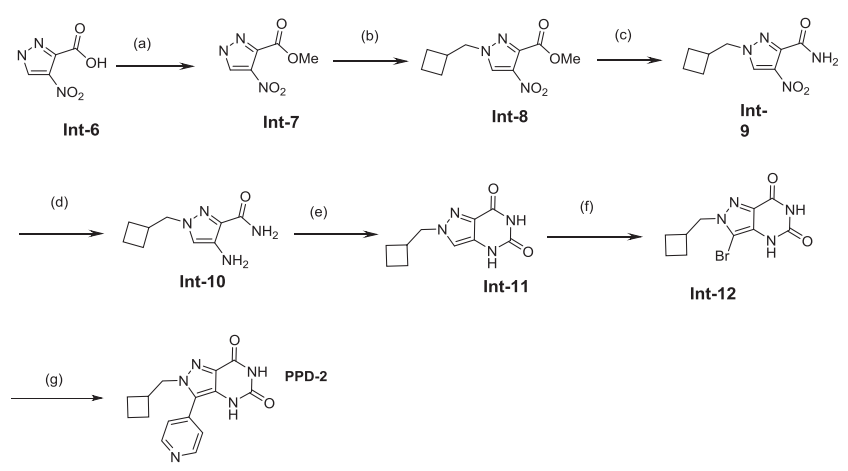

Scheme 2 Synthesis of PPD-2 (a) $\mathrm{CH}_{3} \mathrm{COCl}, \mathrm{MeOH}, 0{ }^{\circ} \mathrm{C}-\mathrm{rt}, 20 \mathrm{~h}$; (b) $\mathrm{K}_{2} \mathrm{CO}_{3}, \mathrm{KI}$, (bromomethyl)cyclobutane, DMF, $60^{\circ} \mathrm{C}, 24 \mathrm{~h}$; (c) $7 \mathrm{M} \mathrm{NH}_{3}$, $\mathrm{MeOH}, 80^{\circ} \mathrm{C}, 8 \mathrm{~h}$; (d) $\mathrm{H}_{2}, 10 \% \mathrm{Pd} / \mathrm{C}, \mathrm{EtOH}, \mathrm{rt}, 8 \mathrm{~h}$; (e) CDI, TEA, MeCN, reflux; (f) $\mathrm{NBS}, \mathrm{AcOH}, \mathrm{MeCN}$, reflux; (g) $\mathrm{Pd}_{2}(\mathrm{dba})_{3}, \mathrm{PCy}_{3}, \mathrm{~K}_{3} \mathrm{PO}_{4}$, dioxane/ water, pyridine-4-boronic acid, reflux, $1.5 \mathrm{~h}$. $\mathrm{rt}$, room temperature.

\section{Generation of PPD analogs}

The chemical syntheses of PPD-1 and PPD-2 are described in Schemes 1 and 2, respectively. The experimental procedures and subsequent characterization of these molecules are summarized in detail in the Supplementary Data. Other PPD analogs were prepared using analogous routes. For PPD-1 (Scheme 1), substituted pyrazole Int-3 was constructed by reaction of diketoester Int-2 with cyclobutylmethyl hydrazine. Nitration of the pyrazole ring, followed by amidation of the ester gave Int-5. Treatment of Int-5 with carbonyldiimidazole under basic conditions gave PPD-1. Compounds 3-11 can be made in a similar fashion by first constructing the appropriately substituted pyrazole ring system. For PPD-2 (Scheme 2), Int-8 was prepared by alkylation of pyrazole Int-7 with bromomethyl cyclobutane. The desired regioisomer was advanced to ring closed product Int-11 using identical conditions as those described for the synthesis of PPD-1. Treatment of pyrazole Int-11 with $\mathrm{N}$-bromosuccinimide gave bromide Int-12, which can be converted to PPD-2 via Suzuki coupling. Analogs $12-19$ can be synthesized similarly by Suzuki coupling of bromide Int12 with the appropriate coupling partner.

\section{RESULTS}

Discovery and antibacterial spectrum of PPD-1

A high-throughput antibacterial screen was conducted to identify compounds that inhibited the growth of PAO1, a wild-type strain of Pseudomonas aeruginosa. PPD-1, a pyrazolopyrimidinedione with MW of 248.3 and ClogP of 1.5 (Figure 1a, inset), was found to be active in this screen, showing $63.8 \%$ inhibition of growth at $98 \mu \mathrm{M}$. Upon hit confirmation, PPD-1 was found to have an $\mathrm{IC}_{50}$ of $46.4 \mu \mathrm{M}$ against wild-type PAO1. Expanded assessment of antibacterial activity revealed the compound was also active against $S$. aureus, demonstrating an $\mathrm{IC}_{50}$ of $20 \mu \mathrm{M}\left(\sim 5 \mu \mathrm{g} \mathrm{ml}^{-1}\right)$ against the laboratory strain RN4220. Although no antibacterial activity was observed up to $100 \mu \mathrm{M}$ vs K. pneumoniae and A. baumannii, PPD-1 had modest activity against laboratory strains of pump-deficient E. coli $\left(\right.$ tolC $^{-}$) with MICs of $\sim 64-128 \mu \mathrm{M}$
Table 1 The MIC (in $\mu \mathrm{g} \mathrm{ml}^{-1}$ ) of PPD-1 antibacterial activity as compared with ciprofloxacin and linezolid against the laboratory S. aureus strain RN4220 and various drug-resistant S. aureus clinical isolates

\begin{tabular}{|c|c|c|c|c|}
\hline $\begin{array}{l}\text { S. aureus } \\
\text { strain }\end{array}$ & Resistance genotype & Ciprofloxacin & Linezolid & $P P D-1$ \\
\hline RN4220 & None & 0.125 & 1 & 8 \\
\hline USA300 & MRSA, quin ${ }^{R}$ & 16 & 1 & 4 \\
\hline SA1900-10 & Bla, MRSA, mecA, macrolide ${ }^{R}$ & $>64$ & 4 & 8 \\
\hline SA1281-07 & MRSA, quin ${ }^{R}, \operatorname{cfr}+\left(\operatorname{lin}{ }^{R}\right)$ & $>64$ & 16 & 8 \\
\hline SA1851-09 & MRSA, quin ${ }^{R}, \operatorname{cfr}+\left(\operatorname{lin}{ }^{R}\right)$ & $>64$ & 8 & 8 \\
\hline SA1609-09 & $\begin{array}{l}\text { MRSA, quin }{ }^{R}, \operatorname{cfr}+\left(\operatorname{lin}{ }^{R}\right) \\
\text { macrolide }^{R}\end{array}$ & $>64$ & 16 & 8 \\
\hline
\end{tabular}

Abbreviations: MRSA, methicillin-resistant Staphylococcus aureus ; PPD, pyrazolopyrimidinedione.

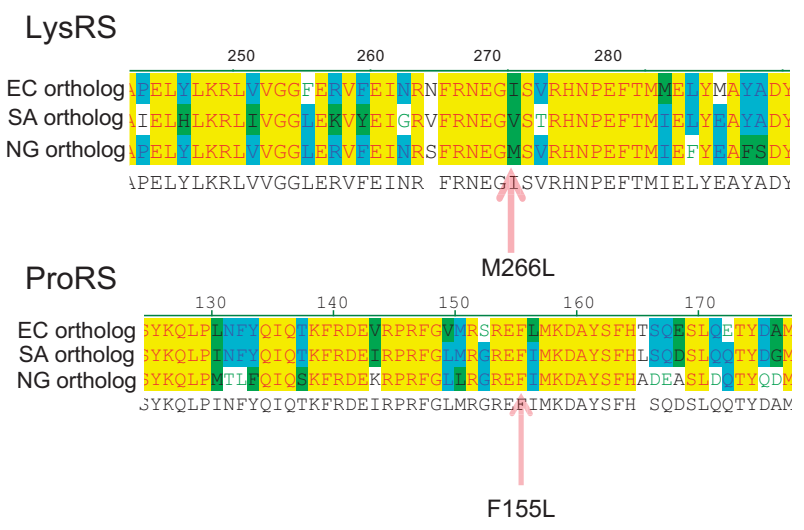

Figure 2 Mutations in distinct tRNA synthetase genes confer resistance to PPD-1 and PPD-2. Resistance to PPD-1 in N. gonorrhoeae (NG) results from a M266L mutation in the active site of lysyl tRNA synthetase (LysRS) (a), whereas resistance to PPD-2 in E. coli (EC) results from a F155L mutation in prolyl tRNA synthetase (ProRS) (b). These targets were subsequently confirmed in tRNA-incorporation assays using purified $S$. aureus (SA) LysRS or E. coli ProRS enzymes (Tables $3 \mathrm{~A}$ and $\mathrm{B}$ ).

$\left(\sim 16-32 \mu \mathrm{g} \mathrm{ml}^{-1}\right)$. PPD-1 was also tested in a standard cytotoxicity (ThlE) assay (Thermo Scientific, Rockville, IL, USA) for its ability to inhibit eukaryotic cell proliferation and was found to have no effect up to $300 \mu \mathrm{M}$ (data not shown). Further assessment of its activity showed that PPD-1 inhibited the growth of a number of multidrug-resistant $S$. aureus strains, including those bearing the $c f r$ plasmid, ${ }^{11}$ which confers resistance to linezolid and other antibiotics (Table 1).

\section{PPD-1 is a protein synthesis inhibitor}

Investigation into the mechanism of action of PPD- 1 began with an evaluation of its ability to inhibit a variety of macromolecular synthesis pathways using the well-established assay in E.coli. PPD-1 was found to specifically inhibit protein synthesis with little-to-no effect on DNA, RNA, cell wall or fatty acid biosynthesis pathways (Figure 1a). PPD-1 was then tested in an E. coli TnT assay and found to be active with an $\mathrm{IC}_{50}$ of $60.7 \mu \mathrm{M}$, which correlates well with its MIC. To further define the point at which PPD-1 inhibits translation, the activity of the compound was compared with other known translation inhibitors in a TnT staging assay, which divides the transcription and translation reaction into biochemically distinct steps (Figure 1b). This assay can distinguish a general protein synthesis inhibitor as either a 
transcription or a translation inhibitor, and indeed, can further classify it as inhibiting earlier or later in the translation process. This is accomplished by adding the inhibitor at 20-25 times the $\mathrm{IC}_{50}$ concentration (effectively shutting down translation) at various times during the TnT assay incubation and measuring how much translation product is made (by measuring the amount of reporter activity) relative to control compounds. The activity of different types of inhibitors (for example, those that inhibit transcription, translation initiation, translation elongation, reporter enzyme inhibition, etc.) can be discerned when reporter activity is graphed as a function of the time of addition of the inhibitor, because the amount of enzyme activity after a given incubation time is inversely proportional to the stage at which the inhibitor acts. In other words, the later in the protein synthesis process that an inhibitor acts, the lower is the reporter activity that is observed (Figure 1b). The activity of PPD-1 in this staging assay was found to correlate closely to that of azithromycin and chloramphenicol, which inhibit peptidyl transferase by binding at different locations on the $50 \mathrm{~S}$ ribosomal subunit. ${ }^{12}$ These data suggested that the mechanism of action of PPD-1 may involve a similar stage of protein synthesis.
Resistance to PPD-1 maps to lysRS

An error-prone PCR platform in Neisseria gonorrhoeae called RATE (for Rapid Antibacterial Target Elucidation) ${ }^{10}$ had been established by our group as one component of our standard mode of action determination strategy, owing to its rapid turnaround time and minimal compound requirement. This approach was therefore used as a first step to genetically identify the molecular target of PPD-1 inhibition. Stable resistant mutants in N. gonorrhoeae were generated that had a fourfold increase in the MIC to PPD- 1 as compared with the parent strain. Sequence analysis showed that the methionine at residue 266 in the active site of lysyl tRNA synthetase (LysRS) had been replaced with a leucine (Figure 2, upper panel), suggesting LysRS was the target of inhibition of PPD-1. To corroborate these results in $S$. aureus, the lysRS gene from $S$. aureus was cloned in the xyloseinducible expression vector pHIS1525 and transformed into $S$. aureus RN4220. Upon induction with $0.5 \%$ xylose, overexpression of LysRS resulted in a fourfold increase in the MIC of PPD-1 (but not other protein synthesis inhibitors) as compared with vector alone (data not shown), suggesting that LysRS is also a target of PPD-1 inhibition in S. aureus. PPD-1 was then tested for its effect on the activity of

Table 2 Structure-activity relationships of PPD analogs

Inactive close-in compounds:

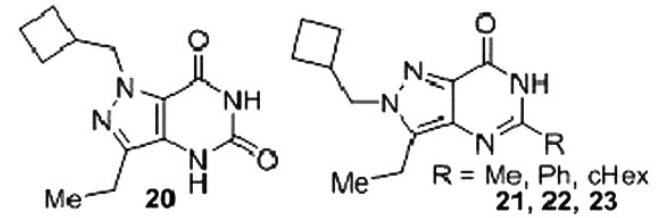

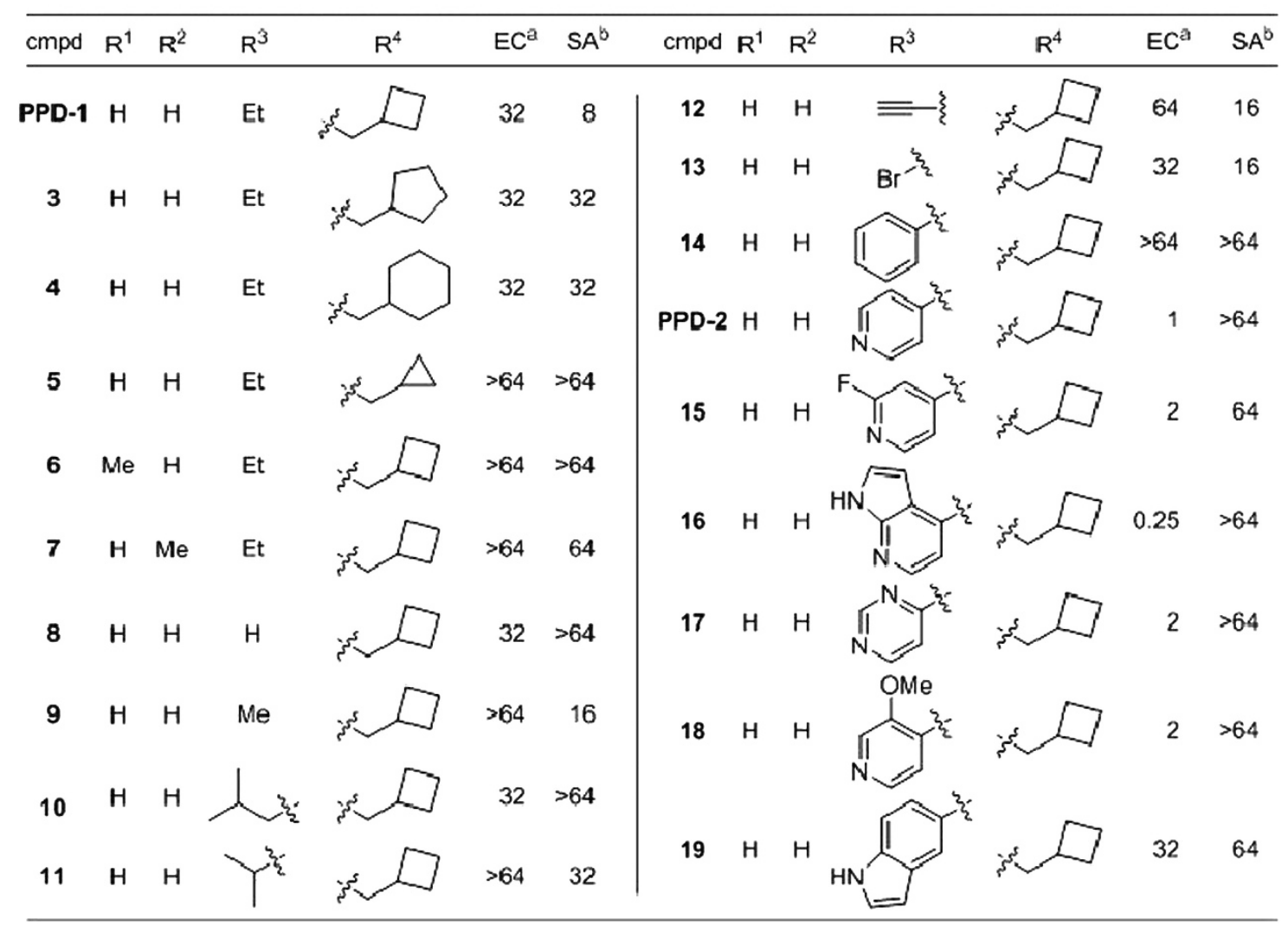


Table 3A Chemical properties and biological activities of PPD-1 and PPD-2 (summary of the chemical properties of PPD-1 and PPD-2)

\begin{tabular}{lcc}
\hline Chemical property & $P P D-1$ & $P P D-2$ \\
\hline MW & 248.13 & 297.12 \\
ClogP & 1.51 & 1.32 \\
ClogD 7.4 & 0.75 & 0.28 \\
TPSA & 84 & 96 \\
\hline
\end{tabular}

Abbreviations: PPD, pyrazolopyrimidinedione; TPSA, topological polar surface area.

purified S. aureus LysRS in vitro and was found to inhibit that enzyme with an $\mathrm{IC}_{50}$ of $21.7 \mu \mathrm{M}$, confirming the resistance mapping and multicopy suppression results.

Structure-activity relationship of pyrazolopyrimidinedione analogs The PPD template is highly amenable to derivatization through a variety of chemical transformations, and various analogs were synthesized and tested (Table 2). Initially, the $\mathrm{R}^{4}$ side chain was varied to larger (compounds 3 and 4) and smaller (compound 5) carbocycles, and while 5- and 6-membered rings were equally active for E. coli, the cyclobutyl derivative PPD-1 proved superior for $S$. aureus. Next, methyl caps were added to the pyrimidinone nitrogens, but compounds 6 and 7 were both inactive. The $\mathrm{R}^{3}$ substituent was removed in compound 8 and varied in size in compounds $\mathbf{9 - 1 1}$, but the ethyl substituent at $R^{3}$ in PPD-1 was the most active for $S$. aureus. A breakthrough in E. coli activity came when heteroaromatic groups (specifically with a nitrogen in the 4 th position) were introduced. A simple pyridine (PPD-2) reduced the E. coli MIC to $1 \mu \mathrm{g} \mathrm{ml}^{-1}$ and pyrrolopyridine 16 had an MIC of $0.25 \mu \mathrm{g} \mathrm{ml}^{-1}$; however, the aryl derivatives (14-19) had reduced $S$. aureus activity. Finally, the pyrazole alkylation regioisomer (compound 20) and some pyrazolopyrimidinones (21-23) were synthesized, but were completely devoid of activity.

As this investigation led to a number of compounds that gained Gram-negative activity at the expense of Gram-positive activity, we selected one of the most potent compounds, PPD-2 (TnT $\mathrm{IC}_{50}=9$ $\mu \mathrm{M})$, for further characterization. Table $3 \mathrm{~A}$ shows a comparison of the chemical properties of PPD-1 and PPD-2.

\section{Resistance to PPD-2 maps to proRS}

The increased Gram-negative antibacterial activity of PPD-2 was exploited to map resistance directly in E. coli using a mutant genomic library. Spontaneously resistant mutants were generated by plating E. coli W3110 tolC::Tn10 on increasing amounts of compound. These stably resistant mutants were generated at a frequency of $3.3 \times 10^{-9}$ at $8 \times$ MIC, and demonstrated an 8-16-fold increase over the MIC of the parent strain. Plasmid libraries were then made from genomic DNA, which was purified from either the sensitive parent strain or a resistant isolate. The library ligations were transformed into W3110 tolC::Tn10 and plated on selective agar containing increasing concentrations of PPD-2. The parental genomic library yielded no colonies, but the resistant mutant genomic library gave two resistant clones at $8 \mu \mathrm{g} \mathrm{ml}^{-1}$ of compound. These plasmids were isolated and retransformed into naive cells to confirm that resistance was plasmid mediated. The two clones were then sequenced and found to have overlapping inserts, both of which containing a mutated proRS (encoding a prolyl tRNA synthetase) with a phenylalanine replacing the leucine at residue 155 (Figure 2, lower panel). The putative target in E. coli was confirmed in vitro using a purified ProRS activity assay, with potent inhibition by PPD-2 observed, corresponding to an $\mathrm{IC}_{50}$ of $0.044 \mu \mathrm{M}$
Table 3B Chemical properties and biological activities of PPD-1 and PPD-2 (relative ability of PPD-1 and PPD-2 to inhibit bacterial growth and various in vitro activity assays)

\begin{tabular}{|c|c|c|}
\hline Biological activity $^{a}$ & $P P D-1$ & $P P D-2$ \\
\hline MIC, S. aureus & 8 & $>64$ \\
\hline $\mathrm{MIC}$, E. coli $\left(\right.$ tolC $\left.\mathrm{C}^{-}\right)$ & 32 & 1 \\
\hline $\mathrm{IC}_{50} \operatorname{TnT}(E$. coll $)$ & 53 & 9 \\
\hline $\mathrm{IC}_{50} \mathrm{TnT}(E$. coli $)+$ excess lysine & 55.5 & 10.75 \\
\hline$I_{50} \operatorname{TnT}(E$. coll $)+$ excess proline & 54.2 & 46.8 \\
\hline$I_{50}$, lysyl tRNA synthetase (S. aur) & 21.7 & 151 \\
\hline$I_{50}$, lysyl tRNA synthetase (S. aur)+excess ATP & 143.4 & 216 \\
\hline$I_{50}$, prolyl tRNA synthetase (E. coli) & 35 & 0.044 \\
\hline$I_{50}$, prolyl tRNA synthetase $(E$. coli $)+$ excess ATP & 90.1 & 0.215 \\
\hline
\end{tabular}

Abbreviations: PPD, pyrazolopyrimidinedione; TnT, transcription-translation.

aValues shown are averages of at least three replicates (shown in $\mu \mathrm{g} \mathrm{ml}^{-1}$ for MICs and $\mu \mathrm{m}$ for TnT and tRNA synthetase assays). Where indicated, excess lysine or proline was added to the TnT assay to a final concentration of $150 \mu \mathrm{m}$. Increasing amounts of ATP was added to the tRNA synthetase assays as described in the text. data shown in the table correspond to the highest amount of ATP added, or $1.5 \mathrm{~mm}$ (12-fold excess).

\section{Preliminary analysis supports differential modes of inhibition by PPD compounds}

As described above and summarized in Table 3B, the relative potency in antibacterial activity for PPD-1 and PPD-2 correlates with their in vitro inhibitory activities. However, although resistance mapping indicated that the primary target of PPD-1 is LysRS, it also demonstrated some activity against ProRS, whereas PPD-2 preferentially inhibited ProRS, suggesting a somewhat divergent mechanism of action. In order to confirm this hypothesis, both compounds were tested in the TnT assay in the presence and absence of excess amounts of either proline or lysine. As shown in Table 3B, the inhibitory activity of PPD-1 in the TnT assay was not affected by addition of either of these amino acids in excess, whereas the activity of PPD-2 decreased significantly by the addition of excess proline but not lysine, which suggests that proline specifically competes with PPD-2 at the active site. The compounds were also evaluated for their ability to compete with ATP in each of the aminoacyl tRNA synthesis assays described above. Addition of excess ATP led to a loss of activity for both compounds against both types of enzymes with the relative effect corresponding to relative potency for each compound (Table 3B). Therefore competition with ATP is likely to have a role in the mechanism of inhibition of both these compounds.

\section{DISCUSSION}

The results presented here describe the identification of a novel series of whole-cell active aminoacyl tRNA synthetase inhibitors using a reverse genomics approach. Although the hit compound, PPD-1, was more potent against $S$. aureus and showed a more balanced activity in the purified LysRS and ProRS assays in vitro, structure-activity relationship studies led to an interesting divergence of activity, with PPD-2 gaining Gram-negative antibacterial potency and submicromolar activity against purified E. coli ProRS. These preliminary results suggest there may be potential within this series to engineer the desired spectrum of activity, potentially both at the target and the species level. In addition to their antibacterial properties, compounds in this series are both 'rule-of-five' compliant, ${ }^{13}$ suggesting the potential for oral bioavailability, and are highly amenable to additional medicinal chemistry investigation. Finally, preliminary computational modeling of the inhibitor binding to the active site (data not shown) suggests additional opportunities for exploration by medicinal chemistry; however, further analysis of this type would be required to 
confirm this hypothesis. Unfortunately, this and any other additional research on this series was not possible given the decision by Pfizer to deprioritize antibacterial discovery in 2011.

There are several reasons why aminoacyl tRNA synthetases have the potential to be good targets for inhibition by novel antibacterial agents $^{14,15}$ and a number of discovery efforts have indeed been conducted around tRNA synthetase inhibitors over the years with varying success. ${ }^{16}$ These enzymes have a pivotal role in protein biosynthesis by catalyzing the transfer of amino acids to their cognate tRNA and therefore are necessary for bacterial growth and survival. Despite their conserved functionality, considerable divergent evolution has resulted in significant structural differences between prokarytic and eukaryotic orthologs, which can be exploited for selectivity. ${ }^{17}$ In addition, a number of natural products, such as albomycin, ${ }^{18}$ indolmycin ${ }^{19}$ or microcin $\mathrm{C}^{20}$ have been shown to kill bacteria via inhibition of tRNA synthetases, suggesting that these enzymes serve as an important Achilles' heel for bacterial survival. Finally, the tRNA synthetases have been clinically validated for Gram-positive skin infections by the topical use of mupirocin (pseudomonic acid) for many decades. ${ }^{21,22}$ However, as for inhibitors of other highly conserved single-gene targets, the potential liability of rapid resistance emergence cannot be overlooked. The most notable recent example was the very promising oxaborole-containing LeuRS inhibitor, GSK2251052, which demonstrated potent preclinical activity against MDR Gram-negative bacteria ${ }^{23}$ and promising exposure and safety in phase I clinical trials. ${ }^{24}$ Unfortunately, facile emergence of resistance in the clinic led to an abrupt termination of phase II studies after several patients developed resistant bacteria during treatment for complicated urinary tract infections. ${ }^{25}$ Because the PPD series described above likely has similar levels of resistance emergence as was observed for GSK2251052 preclinically ${ }^{23}\left(3.3 \times 10^{-9}\right.$ in an E. coli tolC ${ }^{-}$strain for PPD-2 vs 1 in $10^{-7}$ to $10^{-8}$ across all species tested for GSK2251052), overcoming this phenotype would obviously be a key objective in future project advancement. One approach to address this concern would be to design potent inhibition of multiple tRNA synthetases within a single compound, a strategy whose feasibility is supported by the preliminary data presented above.

\section{CONFLICT OF INTEREST}

All co-authors are current or former employees of Pfizer and thereby may own shares in the company.

\section{ACKNOWLEDGEMENTS}

We wish to acknowledge the former leaders of the Pfizer Antibacterial Research Unit for their unfailing dedication and support during very challenging times. We would also like to acknowledge our former colleague, Zuoyu $\mathrm{Xu}$, for development of the staging assay during his time at Pfizer.
1 Boucher, H. et al. Bad bugs no drugs, no ESKAPE. Clin. Infect. Dis. 48, 1-12 (2009).

2 Boucher, H. et al. $10 \times$ ' 20 Progress-development of new drugs active against gramnegative bacilli: an update from the Infectious Diseases Society of America. Clin. Infect. Dis. 256, 1685-1694 (2013).

3 Centers for Disease Control and Prevention Executive Report: Antibiotic Resistance Threats in the United States (2013), http://www.cdc.gov/drugresistance/threat-report2013/.

4 World Health Organization Antimicrobial resistance: global report on surveillance (2014), http://www.who.int/drugresistance/documents/surveillancereport/en/.

5 Silver, L. L. Challenges of antibacterial drug discovery. Clin. Microbiol. Rev. 24 71-109 (2011).

6 Payne, D., Gwynn, M. N., Holmes, D. J. \& Pompliano, D. L. Drugs for bad bugs, confronting the challenges of antimicrobial discovery. Nat. Rev. Drug Discov. 1, 29-40 (2007).

7 Miller, A. et al. Discovery and characterization of QPT-1, the progenitor of a new class of bacterial topoisomerase inhibitors. Antimicrob. Agents Chemother. 52, 2806-2812 (2008).

8 Clinical and Laboratory Standards Institute. Methods for Dilution Antimicrobial Susceptibility Tests for Bacteria that Grow Aerobically 9th edn Vol. 32 (CLSI: Wayne, PA, USA, 2012).

9 Murray, R. W., Melchior, E. P., Hagadorn, J. C. \& Marotti, K. R. S. aureus cell extract transcription-translation assay: firefly luciferase reporter system for evaluating protein translation inhibitors. Antimicrob. Agents Chemother. 45, 1900-1904 (2001).

10 Hartman-Neumann, S., Dunham, S., Gosink, M., Kuhn, M. \& Miller, A. Rapid elucidation of antibacterial targets and resistance determinants using an error-prone genomic library of N. gonorrhoeae, Poster F2-869, Interscience Conference on Antimicrobial Agents and Chemotherapy, Boston, MA, USA (2010).

11 Shen, J., Wang, Y. \& Schwarz, S. Presence and dissemination of the multiresistance gene cfr in Gram-positive and Gram-negative bacteria. J. Antimicrob. Chemother. 68, 1697-1706 (2013).

12 Dunkle, J. A., Xiong, L., Mankin, A. S. \& Cate, J. H. Structures of the Escherichia coli ribosome with antibiotics bound near the peptidyl transferase center explain spectra of drug action. Proc. Natl Acad. Sci. USA 107, 17152-17157 (2010).

13 Pollastri, M. P. Overview of the rule of five. Curr. Protoc. Pharmacol Chapter 9, Unit 9.12 (2010).

14 Lv, I. C. \& Zhu, H. L. Aminoacyl-tRNA synthetase inhibitors as potent antibacterials. Curr. Med. Chem. 19, 3550-3563 (2012).

15 Pohlmann, J. \& Brotz-Osterheltz, H. New aminoacyl tRNA synthetase inhibitors as antibacterial agents. Curr. Drug. Tar. Infect. Dis. 4, 261-272 (2004).

16 Gadakh, B. \& VanAerschot, A. Aminoacyl-tRNA synthetase inhibitors as antimicrobial agents: a patent review from 2006 till present. Expert Opin. Ther. Pat. 22, 1453-1465 (2012).

17 Raczniak, G., Ibba, M. \& Söll, D. Genomics-based identification of targets in pathogenic bacteria for potential therapeutic and diagnostic use. Toxicology 160, 181-189 (2001).

18 Zeng, Y. et al. Biosynthesis of albomycin $\delta 2$ provides a template for assembling siderophore and aminoacyl-tRNA synthetase inhibitor conjugates. ACS Chem. Biol. 7, 1565-1575 (2012).

19 Hurdle, J. G., O'Neill, A. J. \& Chopra, I. Anti-staphylococcal activity of indolmycin, a potential topical agent for control of staphylococcal infections. J. Antimicrob. Chemother. 54, 549-552 (2004).

20 Severinov, K. \& Nair, S. K. Microcin C: biosynthesis and mechanisms of bacterial resistance. Future Microbiol. 7, 281-289 (2012).

21 Ward, A. \& Campoli-Richards, D. M. Mupirocin: a review of its antibacterial activity, pharmacokinetic properties and therapeutic use. Drugs 32, 425-444 (1986).

22 Sutherland, R. et al. Antibacterial activity of mupirocin (pseudomonic acid), a new antibiotic for topical use. Antimicrob. Agents Chemother. 27, 495-498 (1985).

23 Sutcliffe, J. A. Antibiotics in development targeting protein synthesis. Ann. NY Acad. Sci. 1, 122-152 (2011).

24 Tenaro, D. et al. Intrapulmonary pharmacokinetics of GSK2251052 in healthy volunteers. Antimicrob. Agents Chemother. 57, 3334-3337 (2013).

25 Pucci, M. J. \& Bush, K. Investigational antimicrobial agents of 2013. Clin. Microbiol. Rev. 26, 792-821 (2013).

Supplementary Information accompanies the paper on The Journal of Antibiotics website (http://www.nature.com/ja) 